\title{
Efecto de los medicamentos antiepilépticos en el metabolismo de la vitamina $D$ y el impacto negativo relacionado al déficit
}

Effect of antiepileptic drugs in the metabolism of vitamin $D$ and negative impact related to the deficit

$\overline{\text { Juan J. Ramírez-Jiménez MD', Juan M. Alfaro-Velásquez MD² }}$

\begin{abstract}
Resumen: la alteración en el metabolismo de la vitamina $D$ ha ido creciendo en importancia hasta considerarse un problema de salud pública. Cada vez son más frecuentes las etiologías relacionadas al déficit de la vitamina $D$ y con consecuencias a largo plazo, lo que hace necesario conocer no sólo las bases moleculares del metabolismo de la vitamina $D$, con especial atención en la participación de las enzimas de la familia del citocromo P450, sino la descripción de las vías metabólicas, la interacción con el receptor específico, las acciones genómicas y no genómicas, sus resultados en el metabolismo óseo y las acciones extra esqueléticas. Además, la acción directa de los antiepilépticos sobre las enzimas de la citocromo P450 y su efecto negativo sobre los niveles de la vitamina D y el metabolismo óseo. Esta revisión pretende brindar las bases que permitan extrapolar estos conceptos a la práctica clínica e identificar los pacientes con riesgo de hipovitaminosis D debido al uso crónico de antiepilépticos que requieren una conducta terapéutica. Es necesario tener presente que en la actualidad no hay un protocolo clínico universal sobre el seguimiento de estos pacientes incluso con la mejoría en el acceso a recursos diagnósticos.
\end{abstract}

Palabras clave: deficiencia de vitamina D, vitamina D, sistema enzimático del citocromo P-450, receptores de calcitriol, anticonvulsivantes, osteopatía.

Abstract: alteration in vitamin D metabolism has been growing in importance until be considered a
health public problem. Increasingly are more frequent the etiologies related to vitamin D deficiency
and the long-term effects, which making necessary to know not only the molecular basis of vitamin
$D$ metabolism, with special attention to the participation of cytochrome p450 family enzymes, but
also the description of the metabolic pathways, interaction with the specific receptor, genomic and
non-genomic actions, results in bone metabolism and extraskeletal actions. In addition, the direct
action of antiepileptic drugs on cytochrome P450 enzymes and their negative effects on vitamin D
levels and bone metabolism. This review aims to provide the basis that allow to extrapolate these

'Médico Pediatra. Residente de Endocrinología Pediátrica, Universidad de Antioquia-Hospital Universitario San Vicente Fundación (HUSVF). Medellín, Colombia. Correo electrónico: juanramirezjg@hotmail.com

${ }_{2}^{2}$ Médico Pediatra Endocrinólogo. Director Departamento de Endocrinología Pediátrica, Universidad de Antioquia. Medellín, Colombia.

Conflicto de intereses: los autores declaran que no tienen conflicto de intereses

Medicina \& Laboratorio 2015; 21: 131-148

Módulo 13 (Química Clínica), número 14. Editora Médica Colombiana S.A. $2015^{\odot}$

Recibido el 23 de marzo de 2015; aceptado el 16 de abril de 2015

Medicina \& Laboratorio Volumen 21, Números 3-4, 2015. 
concepts to clinical practice and identify patients at risk of vitamin D deficiency due to the chronically use antiepileptic drugs that require therapeutic conduct. It should be remembered that currently there is no universal protocol on clinical monitoring of these patients even with the improvement in access to diagnostic resources.

Key words: vitamin D deficiency, vitamin D, cytochrome P-450 enzyme system, calcitriol receptors, anticonvulsants, osteopathy.

Ramírez-Jiménez JJ. Efecto de los medicamentos antiepilépticos en el metabolismo de la vitamina D y el impacto negativo relacionado al déficit. Medicina \& Laboratorio 2015; 21: 131 - 148.

I a asociación de la vitamina $D$, los anticonvulsivantes y las alteraciones en la salud ósea se Lreconoce desde 1968 [I], siendo particularmente relevante en la niñez, donde se presenta la mayor mineralización ósea. Los niños y adolescentes tratados con medicamentos antiepilépticos presentan alteraciones en el metabolismo óseo asociado a factores de riesgo como la politerapia anticonvulsivante, el uso de medicamentos inductores enzimáticos y la duración de la terapia [2], los cuales incrementan dos a tres veces el riesgo de fracturas [3]. Se ha postulado que este efecto es secundario a los antiepilépticos y la dieta cetogénica, existiendo, además, factores de confusión como la inmovilidad, la desnutrición y la obesidad. Los mecanismos involucrados en la alteración del metabolismo óseo en estos pacientes se han relacionado con múltiples factores endógenos, como la susceptibilidad de cada individuo a tener fracturas, y exógenos, entendidos como los factores de riesgo para fracturas que son modificables, como los ya mencionados, la obesidad, la actividad física, el estadio puberal, el estadio nutricional, entre otros) [4].

Alrededor del 4 I\% de los neurólogos infantiles realiza tamizaje del estatus de la vitamina D y la salud ósea en los pacientes con epilepsia aunque sólo el 9\% formula rutinariamente profilaxis con vitamina D [5]; no obstante, las guías existentes son inconsistentes alrededor del tema y las recomendaciones estándares de La Sociedad Internacional de Densitometría Clínica (ISCD; del inglés, The International Society for Clinical Densitometry) no especifican la epilepsia infantil como una indicación para la realización de la densitometría ósea [6]. Por su parte, la Colaboración Chochrane, en una revisión sistemática de 2005, no encontró suficiente evidencia que soportara el uso rutinario de vitaminas liposolubles en el paciente epiléptico [7]. Entre tanto, la Academia Americana de Pediatría (AAP; del inglés, American Academy of Pediatrics), aunque recomienda que todos los niños deben recibir hasta la adolescencia $400 \mathrm{UI}$ de vitamina D como suplemento, no realizan una recomendación específica de la dosis en los pacientes pediátricos con tratamiento antiepiléptico [8].

Recientemente, una revisión de la literatura realizada por Harijan y colaboradores sobre los marcadores de densidad mineral ósea en los pacientes pediátricos con epilepsia reveló que de 14 estudios de cohorte donde se compara la densidad mineral ósea o la bioquímica ósea en niños tratados con antiepilépticos frente a niños control sanos, siete reportan diferencias significativas, cuatro no encontraron significancia y tres no hacen referencia sobre la significancia estadística de las diferencias entre los grupos. Además, que la prevalencia estimada de déficit de vitamina $D$ en niños tratados con antiepilépticos varía entre 25\% y 75\% [9]. De otro lado, en el único ensayo clínico aleatorizado encontrado en la literatura (hasta nuestro conocimiento), 
realizado por Mikati y colaboradores en 2006, para determinar el impacto de la vitamina D en la densidad ósea en pacientes con terapia anticonvulsiva a largo plazo, se encontró que tanto las dosis de vitamina $\mathrm{D}$ altas (2.000 Ul/día) como las bajas (400 Ul/día) después de un año de tratamiento aumentan la mineralización ósea en la población pediátrica estudiada [10], lo que deja expuestas limitaciones importantes referentes a la duración de la terapia con vitamina D y la falta de control de la dieta/ejercicio [l l ].

La mayoría de los estudios sobre densidad mineral ósea en niños con tratamiento para la epilepsia han encontrado reducciones estadísticamente significativas respecto a los niños sanos [ I l - | 3]. Sin embargo, la mayoría de estos estudios probablemente se encuentren sesgados debido a las muestras pequeñas que no permiten realizar la comparación entre los antiepilépticos específicos o entre los diferentes síndromes epilépticos. Por su parte, los estudios con terapia suplementaria de vitamina $D$ en niños con epilepsia han mostrado limitaciones por la falta de estratificación respecto a los factores que potencialmente influyen en la salud ósea como comorbilidades, nutrición, obesidad y movilidad [14].

A continuación se describen los datos generales sobre la deficiencia de vitamina $\mathrm{D}$ como un problema de salud pública a nivel mundial y se muestran, de forma detallada, los eventos involucrados en su metabolismo para entender de forma adecuada la cascada fisiopatológica que genera resultados negativos en la salud ósea y, por ende, postular algunas conclusiones para que en un futuro se logren establecer estrategias preventivas útiles.

\section{La deficiencia de vitamina $\mathrm{D}$ como problema global}

La prevalencia de la deficiencia de vitamina $D$ es más alta de lo que se ha considerado, incluso en el trópico, donde hay una mejor exposición solar también se ha definido como una epidemia y un problema latente de salud pública [I5]. En países como Colombia se carece de datos estadísticos claros sobre la real magnitud del problema pese a resaltar la presencia de múltiples factores en contra de los adecuados niveles de vitamina D, como la exposición solar limitada, la insuficiente ingesta de vitamina $D$ debido al bajo nivel económico de la población, el color oscuro de la piel, la poca actividad al aire libre, el sedentarismo, la obesidad, el uso de medicamentos que alteran el metabolismo y la lactancia materna sin suplementación.

La deficiencia de vitamina $\mathrm{D}$ es común, con una prevalencia estimada del $9 \%$ en niños americanos en edades entre I y 21 años (tomando como valor de referencia de deficiencia de vitamina D menos de $15 \mathrm{ng} / \mathrm{mL}$ ) [16] y en niños y adultos en Estados Unidos, Canadá, México, Europa y Australia entre el 30\% y el 50\% [ I5]. Por su parte, la Encuesta Nacional de Salud y Nutrición (NHANES, del inglés National Health and Nutrition Examination Survey) indicó que para 2005 y 2006, en Estados Unidos, la prevalencia global de deficiencia de vitamina D en adultos fue del 41,6\%, con mayor proporción en las personas de raza negra e hispanos; además, estableció una prevalencia en los niños de deficiencia de vitamina D del 9\% y de insuficiencia del $61 \%$ [17].

En la población general colombiana no se dispone a la fecha de datos sobre la prevalencia de la hipovitaminosis D y mucho menos del impacto de este problema subclínico y de sus consecuencias a largo plazo; no obstante, la información suministrada en otros estudios puede ser adjudicada a nuestra población, por lo que se considera que Colombia tiene una prevalencia 
importante de déficit de vitamina $D$. Aunque existe controversia sobre cuáles deberían ser los niveles óptimos plasmáticos de vitamina D, Holick y colaboradores determinaron unos valores que hasta la fecha son los más aceptados, en los que se define como deficiencia de vitamina D los niveles plasmáticos de 25-hidroxi vitamina $D(25(\mathrm{OH}) \mathrm{D})$ inferiores a $20 \mathrm{ng} / \mathrm{mL}$ y como insuficiencia los niveles entre $20 \mathrm{ng} / \mathrm{mL}$ y $30 \mathrm{ng} / \mathrm{mL}$ [15]. Los niveles óptimos no asociados a estados patológicos a largo plazo son los mayores de $30 \mathrm{ng} / \mathrm{mL}$, con un amplio rango de normalidad, llegando a los valores de toxicidad por encima de $150 \mathrm{ng} / \mathrm{mL}[|5| 8$,$] .$

\section{La vitamina D más allá de sus funciones esqueléticas}

El metabolito biológicamente activo de la vitamina $D$ es el 1,25-dihidroxi vitamina $D$ $\left(\mathrm{I}, 25(\mathrm{OH})_{2} \mathrm{D}\right)$, cuyo efecto radica en el control del metabolismo esquelético a través de una adecuada interacción de los niveles plasmáticos de calcio/fósforo y en las múltiples funciones extra esqueléticas que le aportan un perfil hormonal. La 1,25-dihidroxivitamina D es erróneamente llamada vitamina, pues actualmente es reconocido que existen formas para que sea sintetizada de manera endógena a través de la exposición solar y a partir del metabolito 7-dihidrocolesterol y la conversión por medio de la familia de la citocromo P450. Es así como la vitamina $D$ ha pasado de ser considerada una vitamina a ser una importante prohormona con múltiples acciones en diferentes tipos de tejidos y procesos fisiológicos, cuya actividad específica está dada por su capacidad de realizar acciones no genómicas (rápidas) y genómicas (lentas), reflejadas en el grupo de actividades clásicas en el hueso, el riñón y el intestino, y en las llamadas actividades no clásicas que no se relacionan con el metabolismo del calcio y el fósforo [19].

Entre las actividades extra esqueléticas de la vitamina $D$ se incluye la secreción de la insulina y su sensibilidad en tejidos periféricos, la función endotelial, la regulación del sistema reninaangiotensina-aldosterona, la modulación de la presión arterial, el control del ciclo celular, la apoptosis y el estrés oxidativo, la reparación del ADN, la actividad antitumoral (colon, próstata, mama, leucemias, carcinoma de células escamosas), la autotolerancia inmunológica, la respuesta inmune ante infecciones, entre otras aún por esclarecer [19].

La deficiencia de vitamina $D$ se ve reflejada por la presencia de raquitismo y osteomalacia, al igual que por el aumento del riesgo de enfermedades crónicas y de alto costo como la diabetes mellitus tipo I y 2, las enfermedades cardiovasculares, que incluyen la hipertensión arterial refractaria al tratamiento, la enfermedad cardioembólica de inicio temprano, la cardiopatía isquémica y la aterosclerosis, y todo tipo de nosologías oncológicas, infecciosas y autoinmunes $[15,19,20]$.

\section{Bases moleculares del metabolismo de la vitamina $D$}

La vitamina D (calciferol) posee una configuración molecular similar a la de los esteroides clásicos (p. ej. cortisol, aldosterona, estradiol), conformada por una estructura de anillo tipo ciclopentanoperhidrofenantreno [20]. Esta vitamina se encuentra en dos formas obtenidas de 
fuentes y precursores diferentes: la vitamina $D_{2}$ (ergocalciferol) y la vitamina $D_{3}$ (colecalciferol). La vitamina $\mathrm{D}_{3}$ se obtiene de dos fuentes principales: a) la dieta, la cual aporta un 10\% de los niveles de vitamina $\mathrm{D}$, a partir del colesterol presente en productos fortificados y aceites de pescado, y b) la producción endógena, que aporta el 90\% restante, mediante la conversión del 7-dehidrocolesterol a previtamina $D_{3}$, la cual por exposición a los rayos ultravioletas del sol (UVB, 290-3I 5 nm) sufre un proceso de isomerización térmica no enzimática formando la vitamina $D_{3}[2 l]$. Entretanto, la vitamina $D_{2}$ no se produce en los humanos, sino que se obtiene a partir del precursor ergosterol proveniente de las membranas celulares de plantas y hongos ingeridos en la dieta y luego de la exposición a rayos UV; sin embargo, la actividad de la vitamina $\mathrm{D}_{2}$ es de un tercio respecto a la vitamina $\mathrm{D}_{3}$ [20].

La vitamina $\mathrm{D}$ no es biológicamente activa por lo que debe sufrir una serie de modificaciones enzimáticas para ejercer su función. Estos procesos son llevados a cabo por las enzimas pertenecientes a la súper familia del citocromo P450 (CYP450), las cuales están formadas por 500 aminoácidos, se encuentran ampliamente distribuidas en el retículo endoplásmico y las mitocondrias, contienen en su estructura un grupo hemo y absorben la luz a 450 nm en estado de reducción, razón por la cual se les dio su nombre. Estas enzimas reciben electrones del estado reducido de la nicotinamida adenina dinucleótido fosfato (NADPH) y realizan sus funciones catalíticas a través de los átomos de hierro (véase figura I) [22].

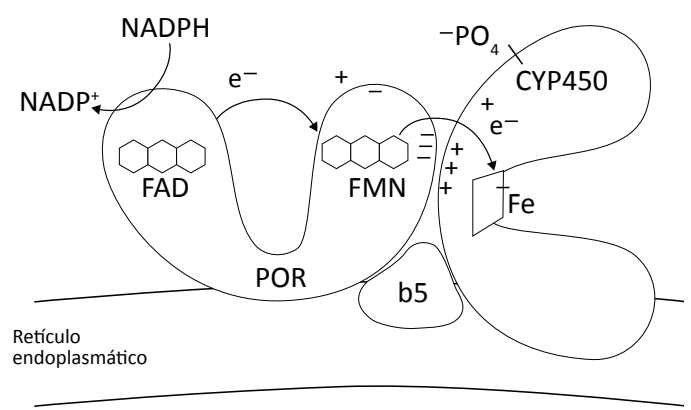

Figura I. Diseño esquemático de la estructura de las enzimas citocromo P450 (CYP450). Tomado y modificado de «The Syndrome of 17,20 Lyase Deficiency» por W.L. Miller, 2012, J Clin Endocrinol Metab, 97, p. 62. Copyright ${ }^{\circledR} 2012$ por The Endocrine Society [22]. NADP= nicotinamida adenina dinucleótido fosfato, $\mathrm{NADPH}=$ estado reducido de la NADP, FAD= Flavín adenín dinucleótido, FMN= Flavín mononucleótido, $\mathrm{POR}=\mathrm{P} 450$ oxidorreductasa, $\mathrm{e}=$ =electrones, $\mathrm{b} 5=$ citocromo $\mathrm{b}_{5}$, CYP450=citocromo $\mathrm{P}^{2} 50, \mathrm{PO}_{4}^{-}=$fosfato, $\mathrm{Fe}=$ hierro.

Una vez formada, la vitamina $D$ es transportada por la proteína plasmática de unión a la vitamina D (DBP; del inglés, vitamin D binding protein) hasta el hígado para que se produzca la primera hidroxilación en el carbono 25 , dando lugar a la 25 -hidroxi vitamina $\mathrm{D}_{3}$ (25 hidroxicolecalciferol). Del grupo de las enzimas citocromo P450, las que participan en el proceso de la 25-hidroxilación son la CYP2RI, la CYP27AI, la CYP3A4 y la CYP2J2, siendo la CYP2RI (25-hidroxilasa) clave central para el adecuado metabolismo(véase figura 2) [23].

La 25-hidroxi vitamina $D_{3}$ es la principal forma circulante de la vitamina $D_{3}$ y es útil como indicador de los niveles de esta vitamina. Después de formada, es transportada por la proteína plasmática de unión a la vitamina $D$ hacia el túbulo proximal del riñón, donde por acción de la enzima mitocondrial CYP27B| ( $\alpha$-hidroxilasa) se produce la segunda hidroxilación en el carbono I $\alpha$, para completar el proceso de activación y convertirse en la forma con función hormonal, conocida como I,25-dihidroxi vitamina $D_{3}$ ( I,25-dihidroxicolecalciferol) (véase figura 2), que ejerce tanto efectos genómicos (respuesta tardía) como no genómicos (respuesta rápida) [20]. 


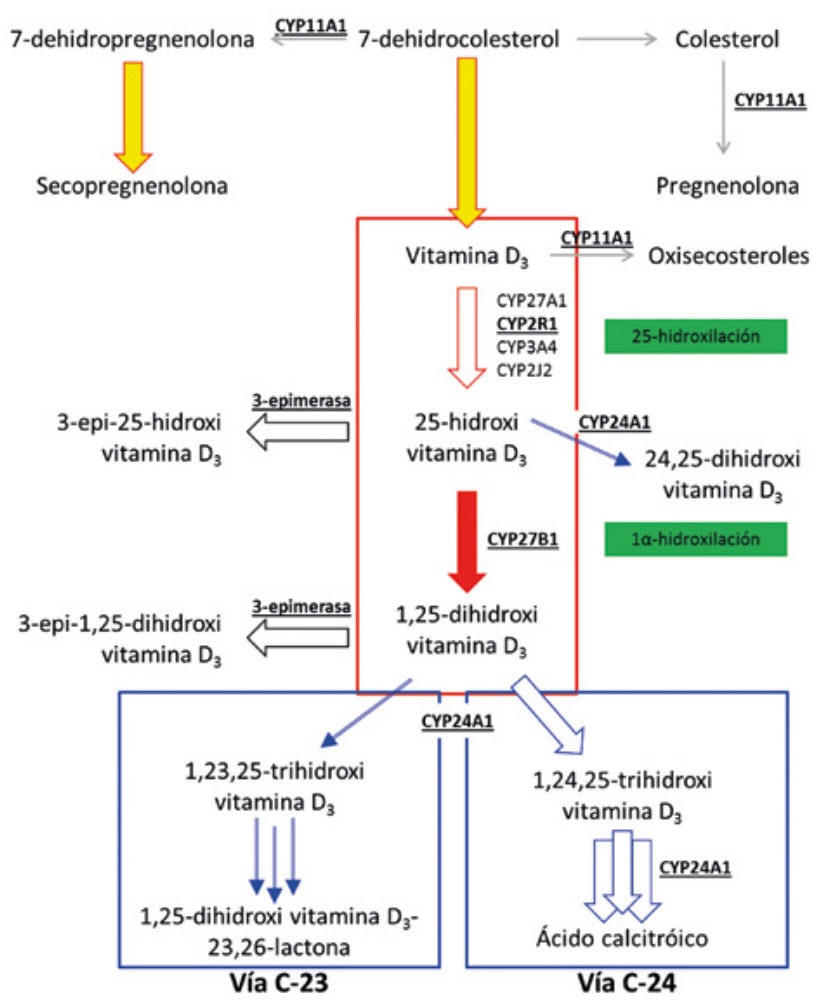

Figura 2. Vías del metabolismo de la vitamina D y la participación de las enzimas citocromo P450 (CYP450). Tomado y modificado de «Cytochromes P450 are essential players in the vitamin D signaling system» por I. Schuster, 20I I, Biochimica et Biophysica Acta (BBA)-Proteins and Proteomics, I8I4, p. 188. Copyright ${ }^{\odot} 2010$ por Elsevier B.V. [24].

La enzima CYP27BI se describió inicialmente en las células de los túbulos renales proximales; sin embargo, recientemente se ha aceptado su presencia en otros sistemas como la placenta, las células monomacrofágicas, la próstata, la mama, el colón, el corazón, el pulmón, el cerebro, la epidermis, las células $\beta$-pancreáticas y las células paratiroides. Esto, sumado a la presencia del receptor de vitamina D (VDR) en dichos sistemas, aporta la posibilidad de producirse localmente la 1,25-dihidroxi vitamina $D_{3}$ donde ejerce una acción autocrina y paracrina [18,24]. De lo anterior se comprenden sus efectos no relacionados con el metabolismo del fósforo y el calcio, y su rol en el control de estos otros sistemas.

El riñón es determinante en la regulación de las acciones de la vitamina $D_{3}$ mediante la acción de la enzima CYP24AI (24-hidroxilasa), que produce el metabolito inactivo 24,25-dihidroxi vitamina $D_{3}$ a partir de la hidroxilación de la 25-hidroxivitamina $D_{3}$, disminuyendo la cantidad disponible de esta para la la-hidroxilación [19]. Además, limita la cantidad de la 1,25-dihidroxivitamina $D_{3}$ en los tejidos blanco mediante su hidroxilación, por dos vías: a) la vía C-24, que conduce a la formación de la 1,24,25-trihidroxi vitamina $D_{3}$, la cual es rápidamente transformada por esta misma enzima a ácido calcitroico, metabolito inactivo de la vitamina $D$ que es excretado por la orina [19,24], y b) la vía C-23, que conduce a la formación de la 1,23,25-trihidroxi vitamina $D_{3}$ que finalmente se convierte en 1,25-dihidroxi vitamina $D_{3}$-23,26-lactona (véase figura 2) [24]. 


\section{Enzimas involucradas en los pasos del metabolismo de la vitamina $D$}

El complejo enzimático del citocromo P450 es fundamental en los procesos de inducción de los metabolitos activos e inactivos de la vitamina $D$ durante las etapas de hidroxilación, por lo que su conocimiento es fundamental para la comprensión de los eventos patológicos que alteran la función de estas enzimas y, por tanto, los niveles de las formas activas de la hormona. Es bien conocido que esta familia participa de forma activa en la farmacocinética de una amplia lista de medicamentos, entre los que se incluyen los antiepilépticos (de todas las generaciones), que inducen la función de estas enzimas, lo que se refleja, a grandes rasgos, en un metabolismo más acelerado de la vitamina $D$, que genera la reducción de los niveles séricos de la 25 -hidroxi vitamina $D_{3}$ y favorece la inducción de enzimas que inactivan la vitamina [24].

La cascada del metabolismo de la vitamina $D$ está regida por el arsenal enzimático de la familia del citocromo P450, que dirige la activación de los precursores inactivos de la vitamina $\mathrm{D}_{3}$ y la degradación de los metabolitos activos por los diferentes procesos de hidroxilación ya descritos (véase figura 2) [24], como se presenta a continuación.

\section{Primera activación: 25 hidroxilación}

Hasta el momento se han descrito cuatro isoformas de las enzimas citocromo P450 involucradas en la primera hidroxilación de la vitamina D: la CYP27AI mitocondrial y tres formas microsomales, la CYP2RI, la CYP2]2 y la CYP3A4, siendo la CYP2RI la que presenta una mayor actividad in vivo debido a su mayor afinidad por la vitamina $D$ (en términos de $K m: 0,45 \mu M$ y 0,67 $\mu$ M para la vitamina $D_{3}$ y $D_{2}$, respectivamente) y su mayor especificidad de sustrato por la vitamina $D$ [25], sin olvidar que un defecto genético de esta enzima es responsable de una forma hereditaria de raquitismo refractario al tratamiento. Las otras tres formas enzimáticas también contribuyen en su función de acuerdo a los niveles de expresión en los diferentes tejidos [26].

La búsqueda sistemática en Amazonia!, un recurso Web que permite la búsqueda de datos del transcriptoma humano, reveló que el perfil de expresión génica de la CYP2RI es amplia, con niveles pico de expresión en el músculo esquelético, la piel, el ovario y el testículo, entre otros. Por su parte, la CYP27AI tiene alta expresión en hígado, donde su principal función es la oxidación de la cadena lateral de los esteroles para producir ácidos biliares, seguido del pulmón, el riñón y el intestino. La CYP2J2 se encuentra de forma importante en hígado, miocardio, placenta, cerebro, en tanto que la CYP3A4 posee una distribución global en los tejidos, pero se expresa principalmente en el hígado y en menor proporción en el intestino [24,27]. La actividad enzimática (en términos de Km y velocidad máxima) de estas enzimas depende del sustrato por el cual tienen afinidad y sus niveles de expresión en los diferentes tejidos de los diferentes agentes que pueden inducirlas o suprimirlas (véase tabla I) [24].

\section{Segunda activación: I $\alpha$-hidroxilación}

El proceso de la l $\alpha$-hidroxilación es llevado a cabo por una sola enzima mitocondrial, la CYP27BI, cuyo principal sitio de actividad es el riñón. Las mutaciones que inactivan el gen de la CYP27BI causan raquitismo dependiente de vitamina D tipo I, una enfermedad autosómica recesiva que responde adecuadamente a la administración de la $\mid \alpha, 25$-dihidroxi vitamina $D$ 


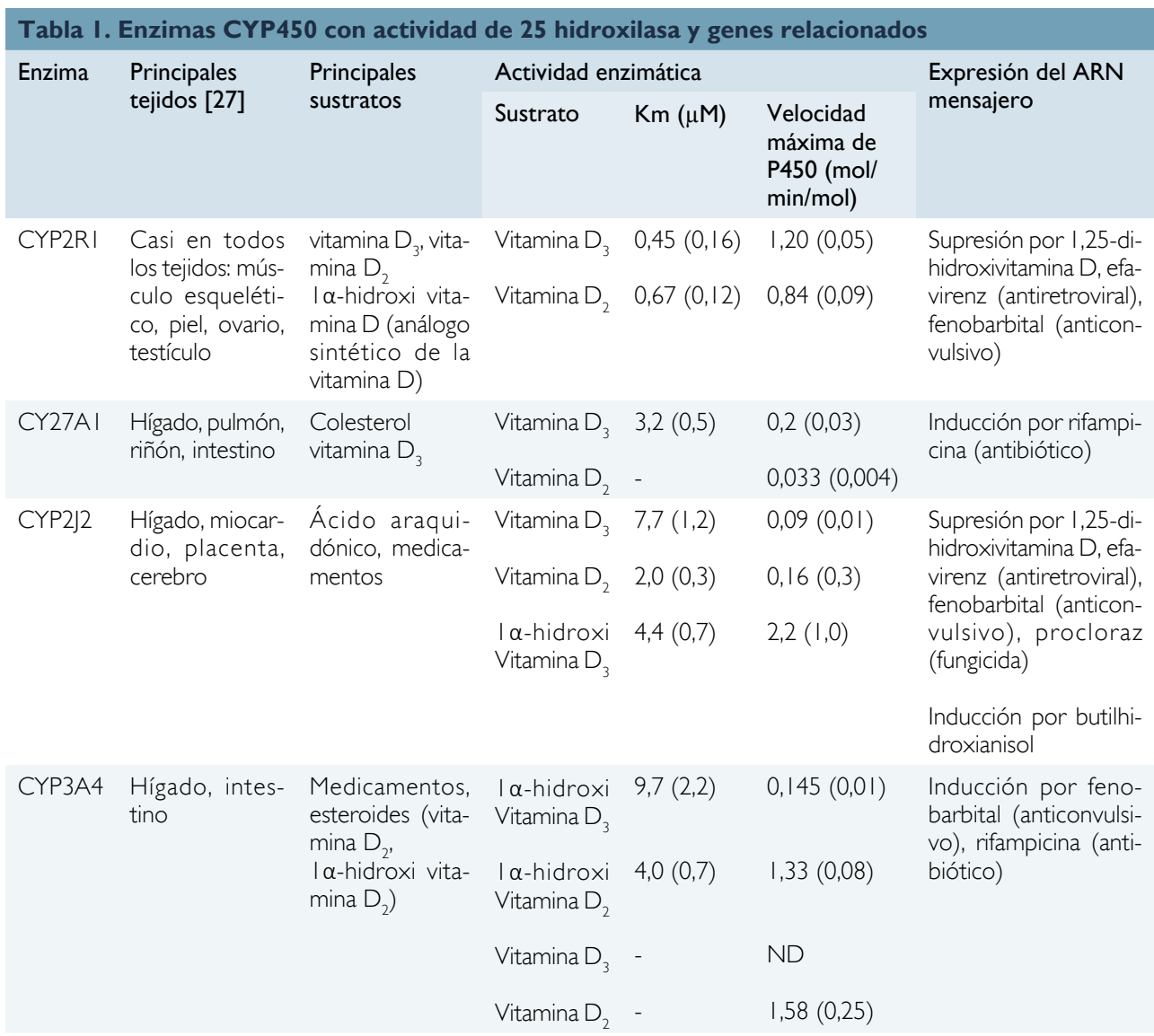

Tomada y modificada de "Cytochromes P450 are essential players in the vitamin D signaling system» por I. Schuster, 201 I, Biochimica et Biophysica Acta (BBA) - Proteins and Proteomics, 1814, p. 189. Copyright ${ }^{\circ} 2010$ por Elsevier B.V. [24].

[28], logrando funciones endocrinas en la homeostasis del calcio, el fósforo y el metabolismo óseo. La regulación de la expresión de la CYP27BI es dependiente de tejido y es dirigida por la hormona paratiroidea (PTH) y la 1,25-dihidroxi vitamina D. En el riñón, ante la presencia de hipocalcemia, se induce la hormona paratiroidea para que estimule la expresión de la CYP27BI, mientras que la I,25-dihidroxi vitamina D ejerce el efecto contrario. Esta regulación se debe a procesos de metilación inducidos por la vitamina $\mathrm{D}$ en el promotor del gen que codifica para la enzima y a la inhibición de la metilación dependiente de la hormona paratiroidea [26].

Los sitios de expresión extrarrenal de la CYP27BI (p. ej. tracto gastrointestinal, páncreas, epidermis, paratiroides, sistema nervioso central, endotelio, tejido adiposo y placenta) favorecen la actividad autocrina y paracrina de la vitamina $D$. En estos tejidos la regulación de la expresión y la actividad de la CYP27B I no depende de los niveles circulantes de la hormona paratiroidea ni la 1,25-dihidroxi vitamina D [24]. En el epitelio respiratorio, por ejemplo, se presentan niveles constitutivos altos de esta enzima, lo que conduce a la producción constante de la 1,25-dihidroxi vitamina D [29], la cual le confiere una actividad antimicrobiana mediante la inducción de la expresión de los genes del péptido de la catelicidina y del correceptor de los receptores 
tipo Toll, el CD 4 [29,30]. De igual manera, en la epidermis la expresión de la enzima es constitutiva, lo que aumenta el metabolismo de la 1,25-dihidroxi vitamina D [31,32], confiriéndole protección contra los efectos nocivos ambientales. Por el contrario, en los macrófagos la expresión de la CYP27BI se encuentra regulada por señales inmunológicas como la producción del interferón- $\gamma$ (INF- $\gamma$ ), lo que aumenta la producción de la I,25-dihidroxi vitamina D que media la respuesta inmune innata frente a patógenos [33,34].

\section{Metabolismo e inactivación de la vitamina $D$}

La forma activa de la vitamina $\mathrm{D}$ tiene un tiempo corto de acción, debido a que induce su propia inactivación a través de la regulación positiva de la enzima CYP24AI, que lleva a cabo los procesos metabólicos que producen los diferentes productos de mayor polaridad y la pérdida de la actividad hormonal [24]. El rol fisiológico de la CYP24AI fue demostrado en un modelo de ratones knock-out para el gen que codifica para esta enzima, que murieron antes de la edad adulta o sufrieron una hipercalcemia severa con osificación intramembranosa causada por un aclaramiento deficiente y retardado de la 1,25-dihidrodi vitamina D en los tejidos [35,36].

La CYP24A I se encuentra localizada en la membrana mitocondrial interna y es expresada en varios tejidos a bajas concentraciones, con alta capacidad de activarse rápidamente en respuesta a los niveles de vitamina $D$ activa. Particularmente, en el hígado esta enzima no se expresa. La activación de la CYP24A I no se produce exclusivamente por la 1,25-dihidroxi vitamina D; también se ha demostrado la inducción por otros compuestos como el ácido litocólico, los ácidos retinoicos, los ligandos del receptor $X$ de pregnano (PXR; del inglés, pregnane $X$ receptor) y por tratamientos prolongados con diferentes grupos de medicamentos antimicrobianos, antituberculosos, anticonvulsivos, entre otros [24].

La CYP24AI participa tanto en el metabolismo de la 25-hidroxi vitamina $D_{3}$ como de la I,25-dihidroxi vitamina $D_{3}$, aunque presenta una unión más estrecha con esta última. Debido al importante rol de la CYP24AI en la modulación de los niveles de la vitamina D activa, la inhibición de esta enzima se convierte en un blanco terapéutico potencial para la prevención de enfermedades como el raquitismo y la osteoporosis. La pobre regulación en los niveles de la CYP24AI contribuye a nosologías que se controlan con la administración de vitamina D, como la enfermedad renal crónica, enfermedades óseas, cáncer, psoriasis, entre otros [15]. En estos casos, la inhibición de la CYP24AI puede ser una estrategia apropiada para incrementar el buen pronóstico.

\section{El receptor de vitamina $D$}

El receptor de vitamina $D$ es de amplia distribución orgánica y ha sido identificado en todas las células nucleadas en diferentes proporciones, lo que confiere un gran espectro de actividad al sistema endocrino vitamina D/receptor de vitamina D. Este receptor hace parte de la súper familia de receptores nucleares y funciona como un factor de transcripción inducido por ligando. Las pocas células que no expresan este receptor son los eritrocitos, los miocitos bien diferenciados y las células de Purkinje del cerebelo [37]; los tejidos que expresan la mayor densidad son el tracto gastrointestinal y el riñón; los que presentan una expresión moderada son la glándula tiroides, el hueso y la piel; y una expresión mínima el hígado [24]. 
La 1,25-dihidroxi vitamina $D$, para ejercer sus acciones no genómicas, se une a los receptores de vitamina $D$ ligados a las caveolas, estructuras de la membrana celular con gran cantidad de lípidos que, una vez activas, producen no sólo la apertura de los canales de calcio y cloro dependientes de voltaje, sino la activación de segundos mensajeros que pueden producir respuestas rápidas de minutos o segundos [37]. El receptor de vitamina D está compuesto por 427 aminoácidos y conformado por cinco estructuras complejas, como se detalla a continuación y se observa en la figura 3 [37,38]:

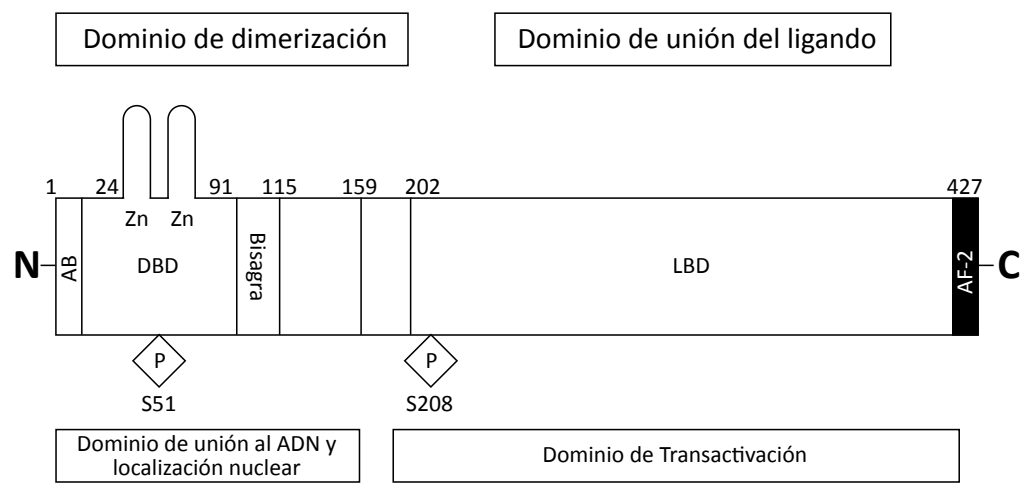

Figura 3. Estructura del receptor de vitamina D (VDR). Tomado y modificado de «Vitamin D and Human Health: Lessons from Vitamin D Receptor Null Mice» por R. Bouillon et al., 2008, Endocrine Reviews, 29, p. 729. Copyright ${ }^{\odot} 2008$ por The Endocrine Society [37]. $\mathrm{DBD}=$ dominio de unión al $\mathrm{ADN}, \mathrm{LBD}=$ dominio de unión al ligando, $\mathrm{AB}=$ dominio $A B, A F-2=$ dominio del factor de activación-2.

$\rightarrow$ Un dominio AB localizado en el extremo amino terminal, de longitud corta y sin función específica. No obstante, se ha encontrado que variantes polimórficas por pérdida de los tres primeros aminoácidos de este dominio incrementan la capacidad de activación del receptor de vitamina D y su interacción con el factor de transcripción IIB (TFIIB) que favorece la expresión génica.

$\rightarrow$ Un dominio de unión al ADN (DBD; del inglés, DNA binding domain), que posee en su estructura dos dedos de zinc necesarios para la activación y la interacción entre el receptor de vitamina $\mathrm{D}$ y los elementos reguladores del ADN y para la translocación del receptor al núcleo. Modelos de ratones knock-out con pérdida de la funcionalidad de uno o los dos dedos de zinc desarrollaron raquitismo resistente y otras manifestaciones como alopecia global. Por su parte, las mutaciones en este dominio se han traducido en niños en raquitismo refractario a la vitamina $\mathrm{D}$.

$\rightarrow$ Una región tipo bisagra de 25 a 50 aminoácidos que le confiere flexibilidad al receptor para la interacción del dominio de unión al ADN con el dominio de unión al ligando y con las proteínas coactivadoras.

$\rightarrow$ Un dominio de unión al ligando (LBD, del inglés, ligand binding domain), conformado por 12 hélices alfa y 3 láminas beta que le aportan gran flexibilidad al receptor. Esta estructura se puede comparar como un bolsillo donde se introduce la 1,25-dihidroxi vitamina $D$ y sus análogos farmacológicos. La fusión entre este dominio y la 1,25-dihidroxi vitamina $D$ induce cambios en la superficie del dominio de unión al ligando necesarios para favorecer 
la heterodimerización con el receptor $X$ de retinoide $(R X R)$. Se han encontrado múltiples mutaciones en este dominio, la más descrita es la de tipo C190W (cistina por triptófano) que causan raquitismo resistente a la vitamina $\mathrm{D}$, debido a que no se produce la interacción con el metabolito activo. Cuando la mutación impide de forma parcial la unión el raquitismo se atenúa y el crecimiento del vello corporal es normal, pero las mutaciones severas generan raquitismo grave y alopecia universal.

$\rightarrow$ Dominio del factor de activación-2 (AF-2), localizado en el extremo carboxilo terminal, el cual modifica la orientación de las 12 hélices alfa del dominio de unión al ligando, activando el receptor de vitamina D y dirigiendo su movimiento desde el citosol al núcleo. Mutaciones en este dominio evitan la translocación del receptor y lo inactivan.

En humanos se han identificado múltiples polimorfismos del gen del receptor de vitamina $\mathrm{D}$, distribuidos a lo largo de toda esta región genética, que están asociados a enfermedades neoplásicas y autoinmunes. El polimorfismo de un solo nucleótido Fokl produce un receptor de vitamina $\mathrm{D}$ más corto por la pérdida de tres aminoácidos, que aunque aún no se ha descrito claramente su impacto clínico se ha relacionado con el cáncer y la diabetes tipo I [37,39].

Después de la unión de la 1,25-dihidroxivitamina $D$ al receptor de la vitamina $D$, este es fosforilado y su conformación en la superficie es reconfigurada, lo que resulta en la liberación de sus correpresores. Posteriormente, se heterodimeriza con el receptor $X$ retinoide $(R X R)$ nuclear y se une a los elementos de respuesta a la vitamina D (VDRE; del inglés, vitamin D response elements), los cuales tienen dos sitios de unión hexaméricos dispuestos en las regiones reguladoras de los genes cuya transcripción es controlada por la vitamina D [37]. Concomitantemente se presenta el reclutamiento de proteínas que alteran el empaquetamiento de la cromatina mediante la modificación enzimática de las histonas (acetilación o metilación), lo que induce el reclutamiento de la ARN polimerasa II para iniciar la transcripción [38].

La modulación de la expresión génica no se produce directamente por la unión del heterodímero receptor de vitamina $D /$ receptor $X$ retinoide con el $A D N$, sino que depende de la habilidad de este complejo para reclutar proteínas correguladoras. Los receptores de vitamina $D$, al igual que otros receptores nucleares, cuando no se encuentran unidos al ligando permanecen transcripcionalmente silenciosos mediante la unión a la cromatina por uno o más correpresores, como el mediador de silenciamiento de los receptores retinoide y de hormonas tiroideas (SMRT; del inglés, silencing mediator of retinoid and thyroid hormone receptors), y el correpresor del receptor nuclear (NCoR; del inglés, nuclear receptor corepressor), que produce la desacetilación de las histonas y, por tanto, mantiene la cromatina empacada de modo denso, haciéndola inaccesible para las proteínas de transcripción [37].

La reorganización del receptor de vitamina D tras la unión al ligando (la 1,25-dihidroxivitamina D) resulta en la unión de las proteínas coactivadoras de las familias CBP/p300 y p I 60, incluyendo los coactivadores de receptores esteroideos (SRC; del inglés, steroid receptor coactivators) como el SRC-I. Estas proteínas tienen una actividad histona acetiltransferasa intrínseca que abre la cromatina, permitiendo la transcripción de los genes regulados por la vitamina $D$, como aquellos involucrados en el metabolismo del fósforo, el calcio y la integridad ósea (p. ej. osteopontina, osteocalcina y calbindina, y canales de calcio como TRPV5 y TRPV6) y los que participan en el control de la proliferación celular, la apoptosis, el estrés oxidativo, la inflamación, la respuesta inmune y la cognición, entre otros [37]. 


\section{Fisiopatología dirigida de los medicamentos antiepilépticos y su impacto en el metabolismo óseo y de la vitamina $D$}

Desde 1968 se ha descrito a los antiepiléticos como un factor de riesgo para la osteopatía, independiente de las demás causas que afectan la masa ósea, debido a que a repercuten en la función neuromuscular y alteran los procesos de mineralización del hueso de forma directa [40]. La edad, el género, así como la dosis y el tiempo de tratamiento, la polifarmacia y la actividad convulsiva que favorece a caídas y fracturas ayudan al desenlace adverso en la estructura ósea. Los antiepilépticos deterioran la calidad de vida del paciente por lo que representan un problema clínico terapéutico. Se entiende que su efecto negativo en el metabolismo óseo no se puede evitar; sin embargo, las medidas que pueden contrarrestarlo como mejorar los estilos de vida tanto físicos como nutricionales y la suplencia de vitamina $D$ no generan el suficiente interés para ser instauradas periódicamente en el tratamiento integral de las convulsiones [4I].

Un mayor entendimiento de los mecanismos fisiopatológicos del déficit óseo asociado al uso de los antiepilépticos puede ayudar al médico a detectar, monitorizar y generar un plan terapéutico en la población vulnerable. Los medicamentos antiepilépticos desarrollan procesos fisiopatológicos que tienen impacto negativo en el hueso y a largo plazo en la densidad mineral ósea. El espectro de manifestaciones puede incluir desde incremento en el recambio óseo sin pérdida del hueso trabecular y cortical hasta osteopenia, osteoporosis y osteomalacia [4I].

Los factores de riesgo asociados al déficit óseo se presentan en ocasiones en un mismo paciente e incluyen: consumo crónico y politerapia, salud ósea alterada previa al inicio del medicamento antiepiléptico, tiempo de estancia hospitalaria y número de hospitalizaciones, edad avanzada, género femenino, actividad física reducida, estado nutricional inadecuado, historia de consumo de alcohol o tabaco y, por último, el uso de medicamentos que alteran el metabolismo óseo ( $p$. ej. esteroides) [5,4I]. Sin embargo, más allá de estos factores de riesgo y si el paciente cursa o no con epilepsia, el solo consumo de los medicamentos antiepilépticos, que con frecuencia son utilizados con otros objetivos terapéuticos, aumenta el riesgo entre dos y tres veces de sufrir fracturas respecto a los individuos que no los consumen [5].

Los mecanismos fisiopatológicos involucrados en la enfermedad ósea inducida por los antiepilépticos incluyen la deficiencia de vitamina $D$ y de calcitonina, los bajos niveles de estrógenos y la hiperhomocisteinemia, entre otros, los cuales pueden estar relacionados entre sí o de forma independiente como se describen en la figura 4 y se profundiza a continuación:

\section{Deficiencia de vitamina D}

La deficiencia de vitamina $D$ es considerada la principal causa de la pérdida de la densidad ósea en los pacientes que consumen medicamentos antiepilépticos, los cuales inducen el sistema enzimático de la citocromo P-450 en el hígado, lo que genera un rápido catabolismo de la vitamina $\mathrm{D}$ y el bloqueo de la primera hidroxilación de la vitamina $\mathrm{D}$, que evita la formación de la 25-hidroxi vitamina $D$. Estos procesos son llevados a cabo por el receptor $\mathbf{X}$ de pregnano (PRX), el cual es activado por los medicamentos antiepilépticos e induce la expresión de la 
CYP24AI, que convierte la 1,25-dihidroxi vitamina $D$ en la forma inactiva soluble 1,24,25-trihidroxi vitamina D, disminuyendo así los niveles de vitamina D biológicamente activos [4I-44].

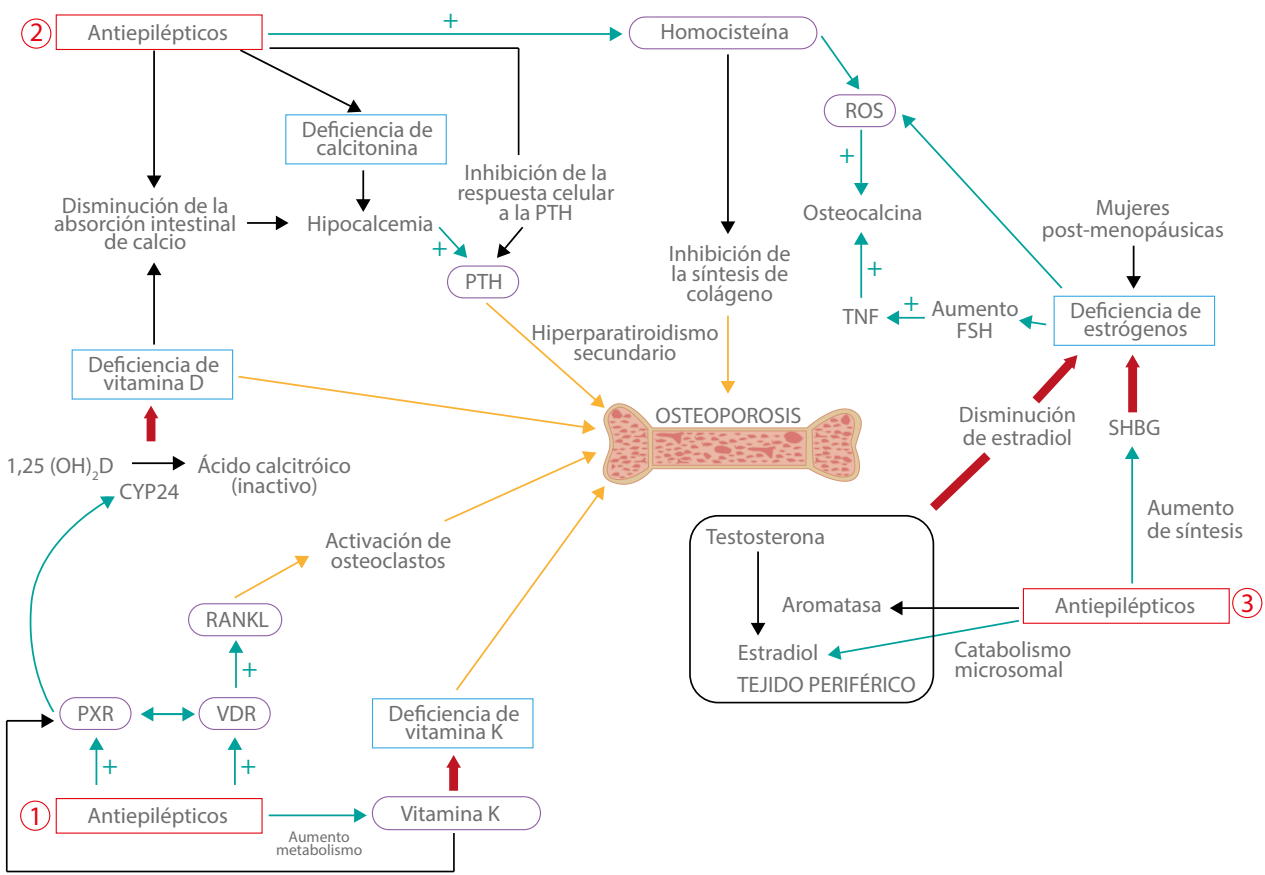

Figura 4. Modelo de fisiopatología de la osteopatía por antiepilépticos convencionales. Tomado y modificado de «lnsights into liaison between antiepileptic drugs and bone» por S. Khanna et al., 2009, Drug Discovery Today, I4, p. 430. Copyright @ 2009 por Elsevier Ltd. [4I]. PXR=receptor $X$ de pregnano, VDR=receptor de vitamina D, RANKL=ligando del receptor activador para el factor nuclear $\kappa B, 1,25(\mathrm{OH})_{2} \mathrm{D}=1,25$-dihidroxi vitamina $\mathrm{D}, \mathrm{PTH}=$ hormona paratiroidea, $\mathrm{ROS}=$ especies reactivas de oxígeno, TNF=factor de necrosis tumoral, $\mathrm{FSH}=$ hormona folículo estimulante, $\mathrm{SHBG}=$ glubulinas fijadoras de hormonas sexuales.

La hiperexpresión de la CYP24AI inducida por el receptor $X$ de pregnano conduce al rápido metabolismo de la forma activa de la vitamina $D$, que causa disminución de sus niveles y, por tanto, las alteraciones en la mineralización de los huesos que dan lugar a la osteomalacia [42]; además, lleva a la pérdida de la protección frente a enfermedades tumorales, por lo que se considera de pobre pronóstico. Incluso, debido a la localización de la CYP24A I en el amplicón $20 \mathrm{q}$ 3,2 es considerada como un oncogen [4I].

También se ha establecido que los antiepilépticos activan al receptor de vitamina $\mathrm{D}$ debido al alto grado de homología que presenta con el receptor $X$ de pregnano. La activación del receptor de vitamina $\mathrm{D}$ conduce a la expresión de proteínas en el osteoblasto como el ligando del receptor activador para el factor nuclear kB (RANKL; del inglés, receptor activator for nuclear factor $\mathrm{kB}$ ligand) que por interacción con el receptor activador para el factor nuclear kB (RANK; del inglés, receptor activator for nuclear factor $\mathrm{kB}$ ) induce la actividad osteoclástica que conlleva al aumento de la remodelación ósea [4I]. 


\section{Hiperparatiroidismo secundario}

La deficiencia de vitamina $D$ conduce a la disminución en los niveles circulantes de calcio y, en compensación, al aumento de los niveles de la hormona paratiroidea, que como resultado produce un hiperparatiroidismo secundario que afectará la salud ósea [4I]. Por un lado, la hormona paratiroidea activa la molécula RANK-L en los osteoblastos favoreciendo la interacción RANK/RANK-L, que se traduce en la activación del osteoclasto y el aumento en la resorción ósea $[45,46]$. Entre tanto, el fenobarbital genera deterioro en la respuesta celular a la hormona paratiroidea, lo que favorece el aumento en la concentración de esta hormona, independiente de los niveles de calcio circulantes [4I].

\section{Deficiencia de calcitonina}

La calcitonina es producida en las células tipo C del folículo tiroideo que actúa fisiológicamente como una antagonista de la hormona paratiroidea a través del bloqueo de sus receptores en el osteoclasto, evitando la resorción ósea. Los medicamentos antiepilépticos, por mecanismos no suficientemente claros, generan una disminución de los niveles circulantes de calcitonina relacionada con la pérdida ósea. Parece ser que los antiepilépticos bloquean de forma directa los receptores sensores de calcio (CAsR) de las células tipo C, impidiendo la liberación de calcitonina de manera independiente de los niveles plasmáticos de calcio [4I,47,48], lo que conduce a la disminución de los niveles de calcitonina y en consecuencia al aumento de los niveles de la hormona paratiroidea, que ejerce los efectos a nivel óseo ya presentados. Por otro lado, la hipocalcemia causada por la terapia con anticonvulsivos podría estar involucrada en los niveles de calcitonina insuficientes [4I].

\section{Déficit de vitamina $K$}

La vitamina $\mathrm{K}$ en su forma de hidroxiquinona es necesaria para los procesos de mineralización ósea mediante la carboxilación de los residuos de glutamato de las proteínas Gla de la matriz ósea, siendo este proceso fundamental para la función de unión de calcio de las proteínas $\mathrm{S}$, Gla y osteocalcina de la matriz ósea preformada. Los antiepilépticos interfieren con el metabolismo de la vitamina $\mathrm{K}$ y reducen los niveles de la forma de hidroxiquinona, lo que genera pobre mineralización de la matriz que se expresa estructuralmente en osteomalacia [4I,49]. Además, la vitamina $K$ se une a los receptores $X$ de pregnano ejerciendo una acción similar a la vitamina $\mathrm{D}$ tras la unión a su receptor, que se traduce en la pérdida ósea cuando disminuyen los niveles de vitamina $\mathrm{K}[4 \mathrm{I}]$.

\section{Agotamiento de los niveles de estrógenos}

En el osteoblasto, la vitamina $\mathrm{D}_{3}$ aumenta la expresión de aromatasa (CYP3A4), la cual cataliza el paso de los esteroides ( 19 carbonos) a estrógenos ( 8 carbonos); estos últimos ejercen una acción paracrina y autocrina en el hueso no sólo induciendo la diferenciación de los osteoblastos, sino también inhibiendo la osteoclastogénesis y promoviendo la apoptosis de osteoclastos [4I]. Los medicamentos antiepilépticos, al generar la disminución de los niveles de vitamina D, producen la reducción en la expresión de la aromatasa, lo que impide que realice los procesos de aromatización, y, en consecuencia, generan un estado de hipoestrogenismo a nivel local [50]; además, aumentan los niveles de las glubulinas fijadoras de hormonas sexuales (SHBG; del inglés, sex hormone-binding globulin), que resulta en la disminución de los esteroides disponibles 
para la formación de los estrógenos, y aumentan los procesos catabólicos de los estrógenos, reduciendo igualmente sus niveles $[4 \mid, 5 \mathrm{I}]$.

El hipoestrogenismo producido conduce a la pérdida de la protección contra moléculas antioxidantes y al aumento en la producción local de la interleucina-7 (IL-7) en el hueso, la cual desencadena eventos inflamatorios como la activación de los linfocitos $T$ con producción de moléculas de interferón (INF) y el aumento de la producción de radicales libres, los cuales en conjunto aumentan los niveles del factor de necrosis tumoral (TNF), producen la liberación de RANK-Ly, por tanto, la inducción de la osteoclastogénesis. Tras un hipoestrogenismo detectado, los altos niveles de la hormona folículo estimulante (FSH) resultantes se relacionan con el aumento de la expresión del factor de necrosis tumoral, el cual tiene un actividad osteoclastogénica importante [4I].

\section{Hiperhomocisteinemia}

Por mecanismos moleculares no claros existe una relación positiva entre el consumo de ácido valpróico y los bajos niveles de ácido fólico, esencial para el metabolismo de la homocisteína. La hiperhomocisteinemia resultante genera alteración de los procesos de entrecruzamiento del colágeno durante la mineralización ósea, favoreciendo la osteoclastogénesis y la producción de radicales libres a nivel local, que interfieren con la masa ósea [52,53].

\section{Antiepilépticos de segunda generación y su efecto en el metabolismo óseo}

En el contexto de las recientes estrategias anticonvulsivas, los medicamentos actualmente disponibles de segunda generación (p. ej. vigabatrina, lamotrigina, topiramato, tiagabina, gabapentina, oxcarbazepina, levetiracetam y zonisamida) tienen un perfil farmacológico más seguro, debido a que presentan menos interacciones medicamentosas, menos efectos adversos $y$, por lo general, no afectan los niveles séricos de calcio [4I]. No obstante, estos medicamentos continúan siendo determinantes negativos en el metabolismo óseo debido a la inhibición de la aromatasa, la inducción del sistema CYP450, el agotamiento de los estrógenos, el antogonismo del folato y la deficiencia de vitamina D, por los mecanismos ya descritos, pero con una menor severidad. Además, han demostrado otros mecanismos fisiopatológicos como la inhibición de la anhidrasa carbónica isoenzima II, la cual está involucrada directamente en los procesos de recambio óseo al aportar bicarbonato y protones como metabolitos necesarios para la resorción ósea $[54,55]$. Los principales mecanismos que afectan el metabolismo óseo de los medicamentos antiepilépticos actuales se describen en la tabla 2 [4I].

\section{Tamizaje y tratamiento de disfunción ósea en pacientes con terapia anticonvulsiva}

Aunque no existen protocolos internacionales para el tamizaje y tratamiento de los pacientes que usan antiepilépticos de forma crónica, evaluar el metabolismo óseo en estos pacientes mediante marcadores bioquímicos de resorción y formación ósea se ha convertido en una estrategia de rutina para establecer una predicción temprana de la disfunción ósea subclínica. La absorciometría de rayos $X$ de doble energía (DEXA; del inglés, dual-energy X-ray absortiometry) es el método de referencia 
para evaluar la densidad mineral ósea debido a que puede detectar hasta pérdidas mínimas (2\%) de mineralización [55], que junto con el estudio bioquímico, que incluye la medición de intermediarios cuantificables como el telopéptido aminoterminal y carboxiloterminal reticulado del colágeno óseo, sialoproteínas óseas, fosfatasa alcalina específica del hueso, la hidroxiprolina y osteocalcina o proteína gla del hueso, entre otros, revela el proceso dinámico del recambio óseo [4I,55].

\begin{tabular}{|c|c|c|c|c|c|c|}
\hline Antiepiléptico & $\begin{array}{l}\text { Inhibición de } \\
\text { la aromatasa }\end{array}$ & $\begin{array}{l}\text { Inhibición de } \\
\text { la anhidrasa } \\
\text { carbónica }\end{array}$ & $\begin{array}{l}\text { Inducción de } \\
\text { la CYP450 }\end{array}$ & $\begin{array}{l}\text { Agotamiento de } \\
\text { los estrógenos }\end{array}$ & $\begin{array}{l}\text { Antagonismo } \\
\text { del folato }\end{array}$ & $\begin{array}{l}\text { Disminución } \\
\text { de vitamina } \\
\text { D e hiperpa- } \\
\text { ratiroidismo } \\
\text { secundario }\end{array}$ \\
\hline Lamotrigina & + & & & \multicolumn{3}{|c|}{+} \\
\hline Levetiracetam & & \multicolumn{5}{|c|}{+} \\
\hline Oxcarbazepina & + & \multicolumn{3}{|c|}{+ (parcial) } & \multicolumn{2}{|r|}{+} \\
\hline Tiagabina & + & & & & & \\
\hline Topiramato & & + & \multicolumn{2}{|l|}{+} & \multicolumn{2}{|r|}{+} \\
\hline Zonisamida & \multicolumn{3}{|c|}{+} & & & \\
\hline \multicolumn{7}{|c|}{$\begin{array}{l}\text { Tomado y modificado de "Insights into liaison between antiepileptic drugs and bone" por S. Khanna et al., 2009, Drug } \\
\text { Discovery Today, I4, p. 430. Copyright (C) } 2009 \text { por Elsevier Ltd. [4 I]. }\end{array}$} \\
\hline
\end{tabular}

A la fecha, y aun conociendo los eventos fisiopatológicos producidos por los antiepilépticos sobre el metabolismo óseo, no todos los neurólogos han incluido los suplementos de calcio y vitamina D en los pacientes en riesgo de disfunción ósea [2]. Incluso todavía permanece el interrogante de si es necesario tomar estas medidas profilácticas tras el uso de los nuevos antiepilépticos, aunque se mantengan los niveles séricos de calcio y vitamina $D$ en el rango normal [56]. Aplicando el modelo paralelo de terapia antiepiléptica tradicional se considera que los pacientes que los consumen hacen parte de la población de riesgo y deben recibir terapia profiláctica [49].

En el año 2007, en la versión 61 de la Reunión Anual de la Sociedad Americana de Epilepsia, se presentaron estudios clínicos que demostraron una prevalencia de osteopatía por antiepilépticos de primera línea (inductores de las CYP450) del 46,2\% y por antiepilépticos de segunda línea (no inductores de las CYP450) del 34,2\% [56], lo que demuestra el beneficio de los antiepilépticos de segunda línea respecto a los de primera línea aunque no de forma estadísticamente significativa.

\section{Conclusiones}

El sistema de la vitamina $D /$ receptor de vitamina $D$ se amplía como un regulador fundamental en una extensa gama de funciones extra esqueléticas, y en ello, diferentes conceptos bibliográficos están de acuerdo en sus efectos beneficiosos en el control de enfermedades diferentes a las esqueléticas. Este sistema es altamente influenciable por diversos factores que pueden impactarlo negativamente y generar enfermedad a largo plazo. En el presente manuscrito se describió cómo los agentes farmacológicos para el tratamiento de la epilepsia utilizados a diario generan eventos caóticos en las vías metabólicas de la vitamina $D$, reduciendo sus valores séricos, que conducen a alteraciones clínicas específicas a nivel óseo y que aumentan el porcentaje de la población vulnerable a otras enfermedades sistémicas. 
Si bien la literatura es clara en demostrar la asociación de causalidad entre el déficit de vitamina $D$ y los agentes antiepilépticos, no se han establecido herramientas terapéuticas claras en beneficio de evitar los desenlaces negativos. El modelo fisiopatológico de la osteopatía por medicamentos antiepilépticos pone en claro el déficit existente de estrategias preventivas universales y es un aliciente para el desarrollo no sólo de métodos a favor del mejoramiento de programas de tamizaje, sino de herramientas terapéuticas para utilizar a favor de la amplia población vulnerable.

\section{Bibliografía}

I. Offermann G, Pinto V, Kruse R. Antiepileptic drugs and vitamin D supplementation. Epilepsia 1979; 20: 3- 15.

2. El-Hajj Fuleihan G, Dib L, Yamout B, Sawaya R, Mikati MA. Predictors of bone density in ambulatory patients on antiepileptic drugs. Bone 2008; 43: 149- 155.

3. Souverein PC, Webb DJ, Petri H, Weil J, Van Staa TP, Egberts T. Incidence of fractures among epilepsy patients: a population-based retrospective cohort study in the General Practice Research Database. Epilepsia 2005; 46: $304-310$

4. Shellhaas RA, Joshi SM. Vitamin D and bone health among children with epilepsy. Pediatr Neurol 20 10; 42: 385-393.

5. Valmadrid C, Voorhees C, Litt B, Schneyer CR. Practice patterns of neurologists regarding bone and mineral effects of antiepileptic drug therapy. Arch Neurol 200I; 58: 1369-1374

6. Baim S, Binkley N, Bilezikian JP, Kendler DL, Hans DB, Lewiecki EM, et al. Official Positions of the International Society for Clinical Densitometry and executive summary of the 2007 ISCD Position Development Conference. J Clin Densitom 2008; I I: 75-91.

7. Ranganathan LN, Ramaratnam S. Vitamins for epilepsy. Cochrane Database Syst Rev 2005: CD004304.

8. Wagner CL, Greer FR. Prevention of rickets and vitamin $\mathrm{D}$ deficiency in infants, children, and adolescents. Pediatrics 2008; I22: I |42-I I52.

9. Harijan P, Khan A, Hussain N. Vitamin D deficiency in children with epilepsy: Do we need to detect and treat it? J Pediatr Neurosci 20 I3; 8: 5- I0.

10. Mikati MA, Dib L, Yamout B, Sawaya R, Rahi AC, Fuleihan $\mathrm{Gel} \mathrm{H}$. Two randomized vitamin D trials in ambulatory patients on anticonvulsants: impact on bone. Neurology 2006; 67: 2005-20I4.

II. Chung S, Ahn C. Effects of anti-epileptic drug therapy on bone mineral density in ambulatory epileptic children. Brain Dev 1994; 16: 382-385.

12. Gniatkowska-Nowakowska A. Fractures in epilepsy children. Seizure 20 0; 19: 324-325.

13. Oner N, Kaya M, Karasalihoglu S, Karaca H, Celtik C, Tutunculer F. Bone mineral metabolism changes in epileptic children receiving valproic acid. J Paediatr Child Health 2004; 40: 470-473.

14. Shellhaas RA, Barks AK, Joshi SM. Prevalence and risk factors for vitamin $D$ insufficiency among children with epilepsy. Pediatr Neurol 20 I0; 42: 422-426.

15. Holick MF. Vitamin D: extraskeletal health. Endocrinol Metab Clin North Am 20 I0; 39: 38 I-400, table of contents.

16. Kumar J, Muntner P, Kaskel FJ, Hailpern SM, Melamed ML. Prevalence and associations of 25-hydroxyvitamin D deficiency in US children: NHANES 200 I-2004. Pediatrics 2009; I24: e362-370.

17. Forrest KY, Stuhldreher WL. Prevalence and correlates of vitamin D deficiency in US adults. Nutr Res 201 I; 31 : 48-54.

18. Adams JS, Hewison M. Update in vitamin D. J Clin Endocrinol Metab 20 I0; 95: 47।-478.

19. Zuluaga Espinosa NA, Alfaro Velásquez JM, Balthazar González V, Jiménez Blanco KE, Campuzano Maya G. Vitamina D: nuevos paradigmas. Medicina \& laboratorio 20I I; 17:21I-246.

20. Norman AW. From vitamin D to hormone D: fundamentals of the vitamin $D$ endocrine system essential for good health. Am J Clin Nutr 2008; 88: 49IS-499S.

21. Christakos S, Ajibade DV, Dhawan P, Fechner AJ, Mady LJ. Vitamin D: metabolism. Endocrinol Metab Clin North Am 20I0; 39: 243-253.

22. Miller WL. The syndrome of 17,20 lyase deficiency. J Clin Endocrinol Metab 20 I2; 97: 59-67.

23. Reichrath J, Lehmann B, Carlberg C, Varani J, Zouboulis CC. Vitamins as hormones. Horm Metab Res 2007; 39: 7I-84.

24. Schuster I. Cytochromes P450 are essential players in the vitamin D signaling system. Biochim Biophys Acta, Proteins Proteomics 201 1; 1814: 186-199.

25. Shinkyo R, Sakaki T, Kamakura M, Ohta M, Inouye K. Metabolism of vitamin D by human microsomal CYP2RI. Biochem Biophys Res Commun 2004; 324: 45I-457.

26. Cheng JB, Motola DL, Mangelsdorf DJ, Russell DW. De-orphanization of cytochrome P450 2RI: a microsomal vitamin D 25-hydroxilase. J Biol Chem 2003; 278: 38084-38093.

27. Le Carrour T, Assou S, Tondeur S, Lhermitte L, Lamb $\mathrm{N}$, Reme T, et al. Amazonia!: An Online Resource to Google and Visualize Public Human whole Genome Expression Data. Open Bioinforma J 20 I0; 4: 5- 10.

28. Sawada N, Sakaki T, Kitanaka S, Kato S, Inouye K. 
Structure-function analysis of CYP27BI and CYP27AI. Studies on mutants from patients with vitamin D-dependent rickets type I (VDDR-I) and cerebrotendinous xanthomatosis (CTX). Eur J Biochem 200 I; 268: 6607-66I 5.

29. Hansdottir S, Monick MM, Hinde SL, Lovan N, Look DC, Hunninghake GW. Respiratory epithelial cells convert inactive vitamin $D$ to its active form: potential effects on host defense. J Immunol 2008; I81: 7090-7099.

30. White JH. Vitamin D signaling, infectious diseases, and regulation of innate immunity. Infect Immun 2008; 76: 3837-3843.

3I. Schuster I, Egger H, Astecker N, Herzig G, Schussler M, Vorisek G. Selective inhibitors of CYP24: mechanistic tools to explore vitamin D metabolism in human keratinocytes. Steroids 200 I; 66: 45I-462.

32. Schuessler M, Astecker N, Herzig G, Vorisek G, Schuster I. Skin is an autonomous organ in synthesis, two-step activation and degradation of vitamin D(3): CYP27 in epidermis completes the set of essential vitamin $\mathrm{D}(3)$-hydroxylases. Steroids 200।; 66: 399-408.

33. van Etten E, Stoffels K, Gysemans C, Mathieu C, Overbergh $\mathrm{L}$. Regulation of vitamin $\mathrm{D}$ homeostasis: implications for the immune system. Nutr Rev 2008; 66: SI 25- 134.

34. Adams JS, Hewison M. Extrarenal expression of the 25-hydroxyvitamin D-I-hydroxylase. Arch Biochem Biophys 2012; 523: 95-102.

35. Prosser DE, Jones G. Enzymes involved in the activation and inactivation of vitamin D. Trends Biochem Sci 2004; 29: 664-673.

36. Masuda S, Byford V, Arabian A, Sakai Y, Demay MB, St-Arnaud R, et al. Altered pharmacokinetics of I alpha,25-dihydroxyvitamin D3 and 25-hydroxyvitamin D3 in the blood and tissues of the 25-hydroxyvitamin D-24-hydroxylase (Cyp24al) null mouse. Endocrinology 2005; 146: 825-834.

37. Bouillon R, Carmeliet G, Verlinden L, van Etten E, Verstuyf A, Luderer HF, et al. Vitamin D and human health: lessons from vitamin $D$ receptor null mice. Endocr Rev 2008; 29: 726-776.

38. Pike JW, Meyer MB, Watanuki M, Kim S, Zella LA, Fretz JA, et al. Perspectives on mechanisms of gene regulation by 1,25-dihydroxyvitamin D3 and its receptor. I Steroid Biochem Mol Biol 2007; 103: 389-395.

39. Audi L, Marti G, Esteban C, Oyarzabal M, Chueca M, Gussinye $M$, et al. VDR gene polymorphism at exon 2 start codon (Fokl) may have influenced Type I diabetes mellitus susceptibility in two Spanish populations. Diabet Med 2004; 21 : 393-394.

40. Petty SJ, Paton LM, O'Brien TJ, Makovey J, Erbas B, Sambrook $\mathrm{P}$, et al. Effect of antiepileptic medication on bone mineral measures. Neurology 2005; 65: I3581365.

4I. Khanna S, Pillai KK, Vohora D. Insights into liaison between antiepileptic drugs and bone. Drug Discov Today 2009; 14: 428-435.

42. Pascussi JM, Robert A, Nguyen M, Walrant-Debray O, Garabedian M, Martin P, et al. Possible involvement of pregnane $X$ receptor-enhanced CYP24 expression in drug-induced osteomalacia. J Clin Invest 2005; I I 5: I77- 86.

43. St-Arnaud R, Arabian A, Travers R, Barletta F, RavalPandya M, Chapin K, et al. Deficient mineralization of intramembranous bone in vitamin D-24-hydroxylase-ablated mice is due to elevated 1,25-dihydroxyvitamin D and not to the absence of 24,25-dihydroxyvitamin D. Endocrinology 2000; 141: 2658-2666.

44. Takasu H, Sugita A, Uchiyama Y, Katagiri N, Okazaki $\mathrm{M}$, Ogata $\mathrm{E}$, et al. c-Fos protein as a target of anti-osteoclastogenic action of vitamin $D$, and synthesis of new analogs. J Clin Invest 2006; I I6: 528-535.

45. Sahota O, Mundey MK, San P, Godber IM, Lawson N Hosking DJ. The relationship between vitamin D and parathyroid hormone: calcium homeostasis, bone tumover, and bone mineral density in postmenopausal women with established osteoporosis. Bone 2004; 35: 312-319.

46. Fox SW, Lovibond AC. Current insights into the role of transforming growth factor-beta in bone resorption. Mol Cell Endocrinol 2005; 243: 19-26.

47. Pack AM. The Association Between Antiepileptic Drugs and Bone Disease. Epilepsy Curr 2003; 3: 91 -95.

48. Qin H, Yang FS. Calcitonin may be a useful therapeutic agent for osteoclastogenesis syndromes involving premature eruption of the tooth. Med Hypotheses 2008; 70: I048-1050.

49. Shearer MJ. Vitamin K. Lancet 1995; 345: 229-234.

50. Szulc P, Chapuy MC, Meunier PJ, Delmas PD. Serum undercarboxylated osteocalcin is a marker of the risk of hip fracture in elderly women. J Clin Invest 1993; 91: 1769- 1774.

5I. Hernandez JL, Garces CM, Sumillera M, FernandezAldasoro EV, Garcia-lbarbia C, Ortiz-Gomez JA, et al. Aromatase expression in osteoarthritic and osteoporotic bone. Arthritis Rheum 2008; 58: 1696-1700.

52. Apeland T, Mansoor MA, Strandjord RE. Antiepileptic drugs as independent predictors of plasma total homocysteine levels. Epilepsy Res 200 I; 47: 27-35.

53. McLean RR, Jacques PF, Selhub J, Tucker KL, Samelson EJ, Broe KE, et al. Homocysteine as a predictive factor for hip fracture in older persons. N Engl J Med 2004; 350: 2042-2049.

54. Casini A, Antel J, Abbate F, Scozzafava A, David S, Waldeck $\mathrm{H}$, et al. Carbonic anhydrase inhibitors: SAR and $X$-ray crystallographic study for the interaction of sugar sulfamates/sulfamides with isozymes I, II and IV. Bioorg Med Chem Lett 2003; I 3: 841-845.

55. De Simone G, Di Fiore A, Menchise V, Pedone C, Antel J, Casini A, et al. Carbonic anhydrase inhibitors. Zonisamide is an effective inhibitor of the cytosolic isozyme II and mitochondrial isozyme V: solution and X-ray crystallographic studies. Bioorg Med Chem Lett 2005; I5: 2315-2320.

56. Kim SH, Lee JW, Choi KG, Chung HW, Lee HW. A 6-month longitudinal study of bone mineral density with antiepileptic drug monotherapy. Epilepsy Behav 2007; 10: 29|-295. 